\title{
Growth and characterization of benzil single crystals using nanotranslation by the modified vertical Bridgman technique
}

\author{
T. Suthan, ${ }^{a}$ P. V. Dhanaraj, ${ }^{a}$ N. P. Rajesh, ${ }^{* a}$ C. K. Mahadevan ${ }^{b}$ and G. Bhagavannarayana ${ }^{c}$ \\ Received 26th July 2010, Accepted 18th March 2011 \\ DOI: 10.1039/c0ce00453g
}

\begin{abstract}
Benzil single crystals have been grown by the modified vertical Bridgman technique using the double wall ampoule with nanotranslation for the first time. The characterization studies of benzil crystals grown by both single and double wall ampoules were analyzed. The grown benzil crystal was confirmed by single crystal and powder X-ray diffraction analyses. Fourier transform infrared analysis confirms the functional groups of the grown benzil. High resolution X-ray diffraction analysis indicates the crystalline perfection of the grown crystals. The UV-Vis-NIR studies show that the grown benzil crystals cutoff wavelength is around $434 \mathrm{~nm}$. The green emission of the grown benzil was identified by photoluminescence studies. The thermal property of the grown benzil was studied by thermogravimetric and differential thermal analyses. The dielectric measurements of benzil crystals were carried out with different frequencies and temperatures and the results indicate an increase in dielectric and conductivity parameters with the increase of temperature at all frequencies. The second harmonic conversion efficiency of the grown benzil was determined.
\end{abstract}

\section{Introduction}

The increasing demand on organic materials for technological applications has prompted researchers to look for newer promising materials..$^{1-3}$ Nonlinear optical (NLO) materials have been attracting a great deal of interest due to their applications such as high speed information processing, optical communications, optical electronics and optical data storage., ${ }^{4,5}$ Moreover the microelectronics industry needs replacement of dielectric materials in multilevel interconnected structures with new low dielectric constant $\left(\varepsilon_{\mathrm{r}}\right)$ materials. ${ }^{6}$

Benzil $\left(\mathrm{C}_{14} \mathrm{H}_{10} \mathrm{O}_{2}\right)$ is a non-hygroscopic organic material with good NLO properties. ${ }^{7}$ Growth and studies of benzil single crystals using different crystal growth techniques are available in the literature. Solution grown benzil single crystal has very slow growth rate compared to the melt method and its structural perfection and crystal quality are low. ${ }^{8}$ Lan and Song have grown a benzil crystal by a vertical dynamic gradient freeze technique and studied its optical properties. ${ }^{9}$ In the Czochralski technique, it is very difficult to stabilize the growth of benzil. ${ }^{10-12}$ In the Bridgman technique, benzil crystals were grown by using single wall ampoules. ${ }^{7,12,13}$ As better quality single crystals are needed for device application, it is necessary to improve the method of growth. An effective control of growth environments is very

${ }^{a}$ Centre for Crystal Growth, SSN College of Engineering, Kalavakkam, 603110, India. E-mail: rajeshnp@ssn.edu.in; Fax: +91 044 27474844; Tel: +91044 27474844; rajeshnp@hotmail.com

${ }^{b}$ Physics Research Centre, S.T. Hindu College, Nagercoil, 629 002, India ${ }^{c}$ C.G.C. Section, National Physical Laboratory, New Delhi, 110 012, India much necessary. In order to achieve this, in the present study, we have made an attempt to grow benzil single crystals using a double wall ampoule with nanotranslation by the modified vertical Bridgman technique. For comparison purposes, benzil single crystals were also grown using the conventional single wall ampoule. The grown crystals were characterized by carrying out single crystal X-ray diffraction (XRD), powder XRD, Fourier transform infrared (FTIR), high resolution X-ray diffraction (HRXRD), UV-Vis-NIR, photoluminescence (PL), thermal, dielectric and second harmonic generation (SHG) studies.

\section{Experimental}

Benzil single crystals were grown by the modified vertical Bridgman technique using the single zone furnace. For translation purposes, a nano-stepper translation assembly was used as reported in our previous paper. ${ }^{14}$ Here we have grown benzil crystals by using two types of ampoules. First one is the single wall ampoule (Fig. 1(a)) and second one is the double wall ampoule with cone angle (Fig. 1(b)). In the Bridgman technique, the conical shape of the ampoule is an important parameter, since conical tip in the ampoule is the point of initiation of the solidification and controls further growth. ${ }^{15}$ Researchers reported that the ampoules with cone angles in the range $15-24^{\circ}$ are found to be the most optimum to obtain inclusion-free good quality transparent crystals. ${ }^{16-18}$ Here we selected the cone angle of $19-23^{\circ}$ for the double wall ampoule for the growth of benzil single crystals.

For crystal growth, both single and double wall ampoules filled with benzil (Sigma Aldrich) were evacuated to $10^{-4}$ Torr 


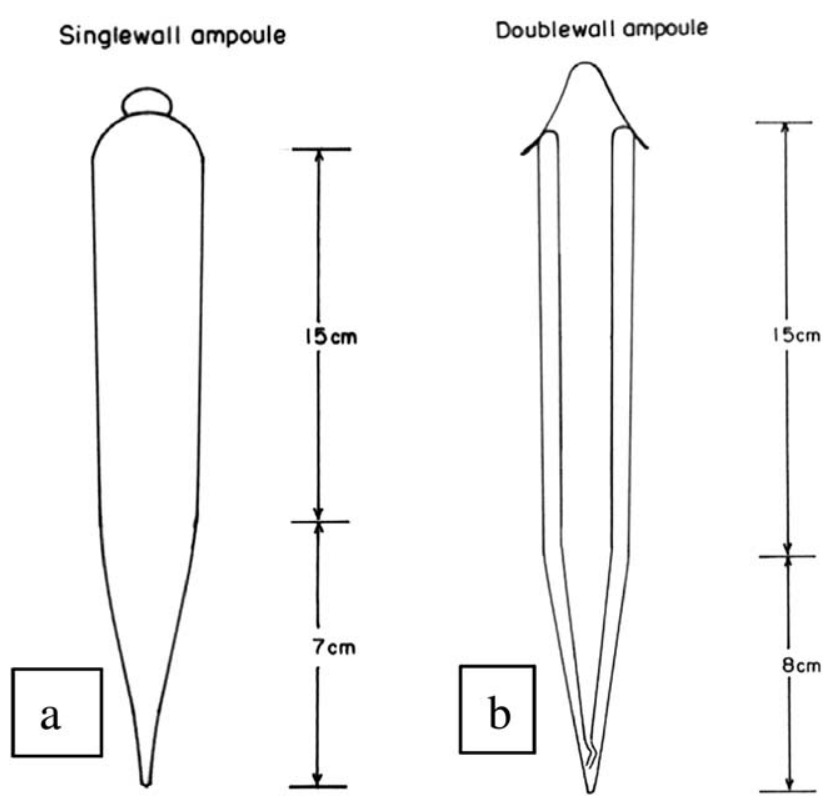

Fig. 1 Schematic of the (a) single wall ampoule and (b) double wall ampoule.

and then sealed off. The temperature in the upper part of the furnace was maintained close to the melting point of the benzil. The material melted in the hotter region and the temperature was kept constant, the melt was homogenized for $24 \mathrm{~h}$ to avoid bubble formation during crystal growth. Here growth was initiated by moving the ampoule from the hot zone to the cold zone very slowly with a translation rate of $0.03 \mathrm{~mm} \mathrm{~h}^{-1}$ to initiate lesser number of nuclei in the outer tube of the ampoule. The substance after melting fills the annular space between the inner and outer tubes and acts as an insulator to prevent thermal shocks entering the tube during the growth. The space above the melt level also acts as a thermal insulator due to vacuum. As the tip of the ampoule passed the interface, the crystallization of the molten benzil was initiated by self-nucleation and the crystal growth was started and continued through the length of the melt. After growth the heating profile was reduced slowly at a rate of $1{ }^{\circ} \mathrm{C} \mathrm{h}^{-1}$ in order to avoid cracks due to the difference in the thermal expansion coefficient between the glass and crystal. The as grown benzil single crystals using single and double wall ampoules are shown in Fig. 2(a) and (b) respectively. The crystals were removed from the ampoules carefully by using a standard diamond wheel cutter. Fig. 2(c) shows the cut and polished portion of the crystal grown by the double wall ampoule.

\section{Results and discussion}

\subsection{X-Ray diffraction studies}

Single crystal X-ray diffraction data of the benzil crystal were collected by using an Enraf Nonius CAD4 single crystal X-ray diffractometer with $\mathrm{MoK} \alpha$ radiation in the wavelength of $0.717073 \AA$ to identify the structure and estimate the lattice parameter values. At the room temperature the observed lattice parameters are shown in Table 1. The grown benzil belongs to the hexagonal system with noncentrosymmetric space group
$P 3_{1} 21$. The obtained cell parameters are in good agreement with the JCPDS file. ${ }^{19}$

Powder X-ray diffraction study was carried out by employing an XPERT-PRO diffractometer with $\mathrm{CuK} \alpha(\lambda=1.54056 \AA)$ radiation using a tube voltage and a current of $40 \mathrm{kV}$ and $30 \mathrm{~mA}$ respectively. The powdered sample was scanned over the range $10-70^{\circ}$ at the rate of $1^{\circ} \mathrm{min}^{-1}$. The powder X-ray diffraction pattern of the grown crystal is shown in Fig. 3. The positions of the peaks are in good agreement with the JCPDS file. ${ }^{19}$

\subsection{Fourier transform infrared (FTIR) analysis}

The FTIR spectrum of benzil was recorded by a Perkin-Elmer FTIR Spectrum RXI Spectrometer using the KBr pellet technique in the frequency range $400-4000 \mathrm{~cm}^{-1}$ to identify the presence of functional groups in the grown crystal. Fig. 4 shows the FTIR spectrum of the benzil single crystal. The intense broad peak at $3431 \mathrm{~cm}^{-1}$ is due to $\mathrm{O}-\mathrm{H}$ stretching. The absorption peaks at 3062 and $2823 \mathrm{~cm}^{-1}$ are assigned to aromatic $\mathrm{C}-\mathrm{H}$ stretching. ${ }^{20}$ Highly intense and well defined sharp peaks observed at 1660 and $1590 \mathrm{~cm}^{-1}$ are due to the $\mathrm{C}=\mathrm{O}$ stretching vibration of the ketone group. ${ }^{20}$ The peaks at 871,788 and $716 \mathrm{~cm}^{-1}$ are assigned to aromatic $\mathrm{C}-\mathrm{H}$ bending.

\subsection{HRXRD studies}

The crystalline perfection of the grown benzil single crystals was characterized by HRXRD by employing a multicrystal X-ray diffractometer developed at National Physical Laboratory (NPL). ${ }^{21}$ The well-collimated and monochromated $\mathrm{MoK} \alpha_{1}$ beam obtained from the three monochromator $\mathrm{Si}$ crystals set in dispersive $(+,-,-)$ configuration has been used as the exploring $\mathrm{X}$-ray beam. The specimen crystal is aligned in the $(+,-,-,+)$ configuration. Due to the dispersive configuration, though the lattice constants of the monochromator crystal(s) and the specimen are different, the unwanted dispersion broadening in the diffraction curve (DC) of the specimen crystal is insignificant. The specimen can be rotated about the vertical axis, which is perpendicular to the plane of diffraction, with a minimum angular interval of 0.4 arc s. The DC was recorded by the socalled $\omega$ scan wherein the detector was kept at the same angular position $2 \theta_{\mathrm{B}}$ with wide opening for its slit.

Fig. 5(a) shows the high-resolution diffraction curve (DC) recorded for a typical single wall ampoule grown benzil single crystal specimen using the (110) symmetrical Bragg geometry by employing the multicrystal X-ray diffractometer described with $\mathrm{MoK} \alpha_{1}$ radiation. The solid line (convoluted curve) is well fitted with the experimental points represented by the filled circles. On deconvolution of the diffraction curve, it is clear that the curve contains an additional peak, which is 32 arc s away from the main peak. This additional peak depicts an internal structural very low angle (tilt angle $\leq 1$ arc min) boundary ${ }^{22}$ whose tilt angle (misorientation angle between the two crystalline regions on both sides of the structural grain boundary) is $23 \mathrm{arcs}$ from its adjoining region. The FWHM (full width at half maximum) of the main peak and the very low angle boundary are respectively 24 and 34 arc s. Though the specimen contains a very low angle boundary, the relatively low angular spread of around 200 arc $\mathrm{s}$ of the diffraction curve and the low FWHM values show 

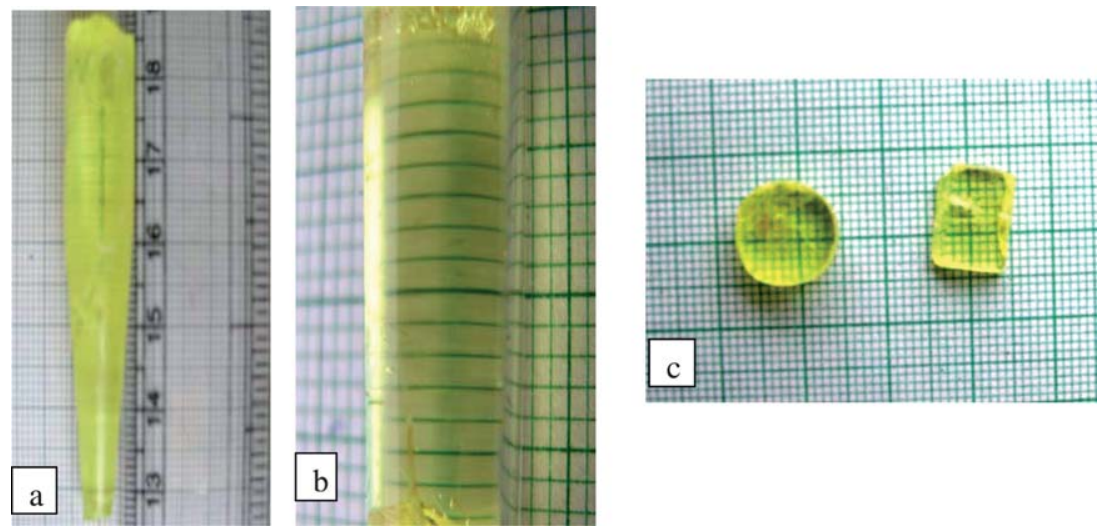

Fig. 2 Photographs of the (a) single wall ampoule grown crystal, (b) double wall ampoule grown crystal, and (c) cut and polished portion of the double wall ampoule grown crystal.

Table 1 Single crystal XRD data of benzil

\begin{tabular}{lll}
\hline Parameter & Reported19 & Present study \\
\hline$a$ & $8.410 \AA$ & $8.44 \AA$ \\
$b$ & $8.410 \AA$ & $8.44 \AA$ \\
$c$ & $13.68 \AA$ & $13.72 \AA$ \\
$\alpha$ & - & $90^{\circ}$ \\
$\beta$ & - & $90^{\circ}$ \\
$\gamma$ & - & $120^{\circ}$ \\
System & Hexagonal & Hexagonal \\
\hline
\end{tabular}

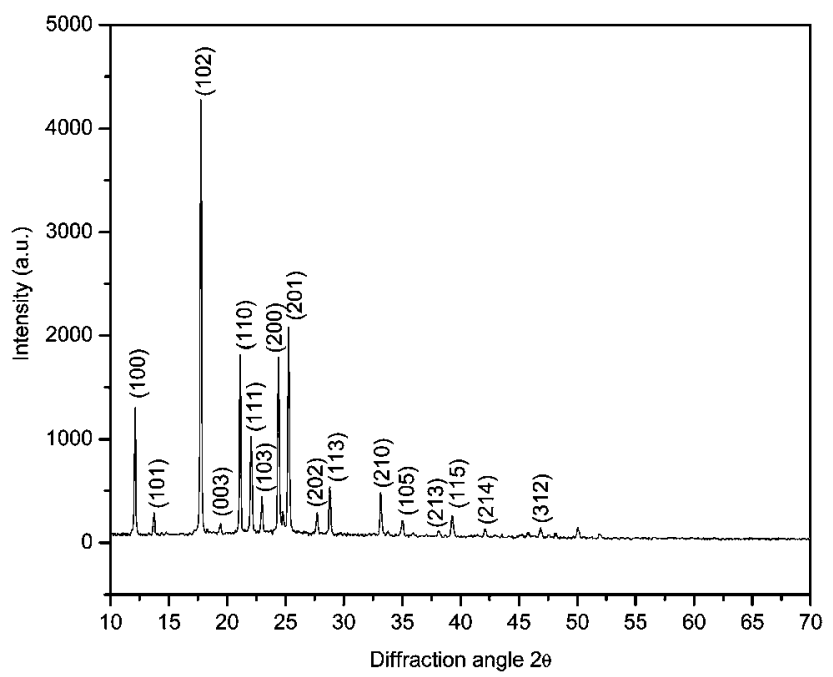

Fig. 3 Powder X-ray diffraction pattern of benzil.

that the crystalline perfection is reasonably good. Thermal fluctuations or mechanical disturbances during the growth process could be responsible for the observed very low angle boundary. It may be mentioned here that such very low angle boundaries (which may hardly deteriorate the properties) could be detected with well resolved peaks in the diffraction curve only because of the high-resolution of the multicrystal X-ray diffractometer used in the present studies.

Fig. 5(b) shows the high-resolution diffraction curve (DC) recorded for a typical double wall ampoule grown benzil single

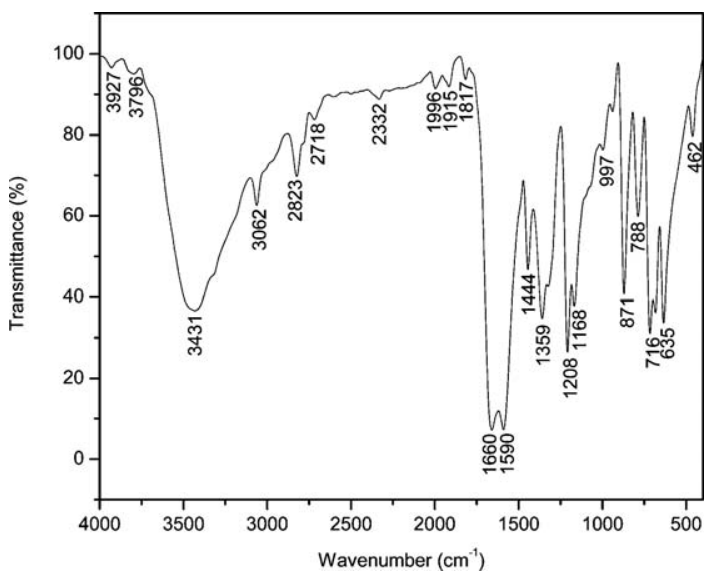

Fig. 4 FTIR spectrum of benzil.

crystal specimen using (110) diffracting planes in the symmetrical Bragg geometry by employing the multicrystal X-ray diffractometer with $\mathrm{MoK} \alpha_{1}$ radiation. The solid line (convoluted curve) is well fitted with the experimental points represented by the filled circles. On deconvolution of the diffraction curve, it is clear that the curve contains two additional peaks, which are 24 and 18 arc $\mathrm{s}$ away from the main peak. These two additional peaks correspond to two internal structural very low angle boundaries whose tilt angles are 24 and 18 arc s from their adjoining regions. The FWHM of the main peak is 20 arc s and that of both the low angle boundaries is 25 arc s. Though the specimen contains very low angle boundaries, the relatively low angular spread of around 100 arc $\mathrm{s}$ of the diffraction curve and the low FWHM values show that the crystalline perfection is reasonably good. Thermal fluctuations or mechanical disturbances during the growth process could be responsible for the observed very low angle boundary. Considering the ampoules used in the growth of benzil crystals in the present study, the main peak observed becomes sharper when the single wall ampoule is replaced by the double wall ampoule. This indicates an improvement in the quality of the benzil crystal grown by using the double wall ampoule. 

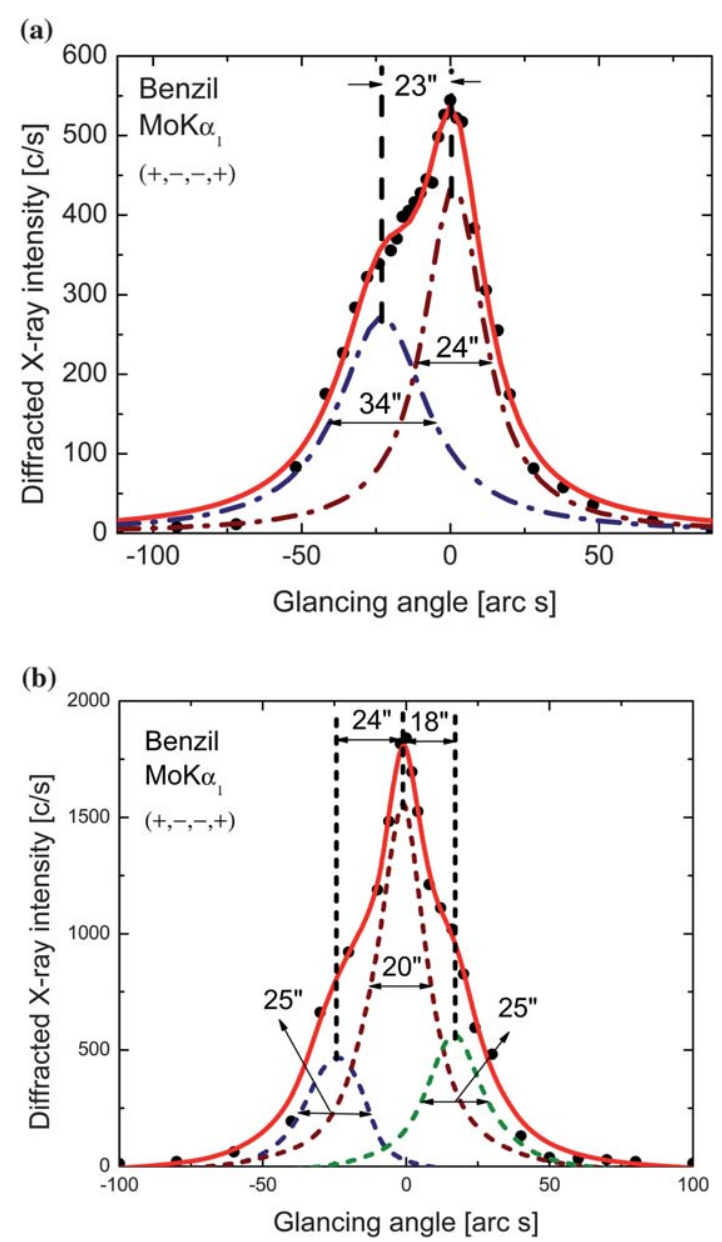

Fig. 5 (a) High-resolution X-ray diffraction curve recorded for a typical single wall ampoule grown benzil single crystal using (110) diffracting planes in the symmetrical Bragg geometry with $\mathrm{MoK} \alpha_{1}$ radiation. (b) High-resolution X-ray diffraction curve recorded for a typical double wall ampoule grown benzil single crystal using (110) diffracting planes in the symmetrical Bragg geometry with $\mathrm{MoK} \alpha_{1}$ radiation.

\subsection{Thermal properties}

Thermogravimetric (TG) and differential thermal analyses (DTA) of benzil single crystals were carried out using a SDT Q600 simultaneous thermal analyzer in the temperature range $30-300{ }^{\circ} \mathrm{C}$ in inert nitrogen atmosphere at a heating rate of $10^{\circ} \mathrm{C}$ $\mathrm{min}^{-1}$. A platinum crucible was used for heating the sample. The results obtained from TG and DTA are shown in Fig. 6. TG analysis shows that the material exhibits single sharp weight loss starting at $145{ }^{\circ} \mathrm{C}$ and below this temperature no significant weight loss is observed. The DTA shows a sharp endothermic peak observed at $96{ }^{\circ} \mathrm{C}$ which is assigned to the melting point of the grown crystal at which no weight loss in TGA has been noticed and another sharp endothermic peak observed at $226^{\circ} \mathrm{C}$ which is assigned to decomposition. Before melting no characteristic exothermic or endothermic peaks were observed. It is observed that no phase transition or decomposition occurs up to the melting point. Sharpness of the endothermic peaks observed in DTA indicates a good degree of crystallinity of the sample. The melting and decomposition points of the grown crystal were also confirmed by using a MONATCH melting point apparatus.

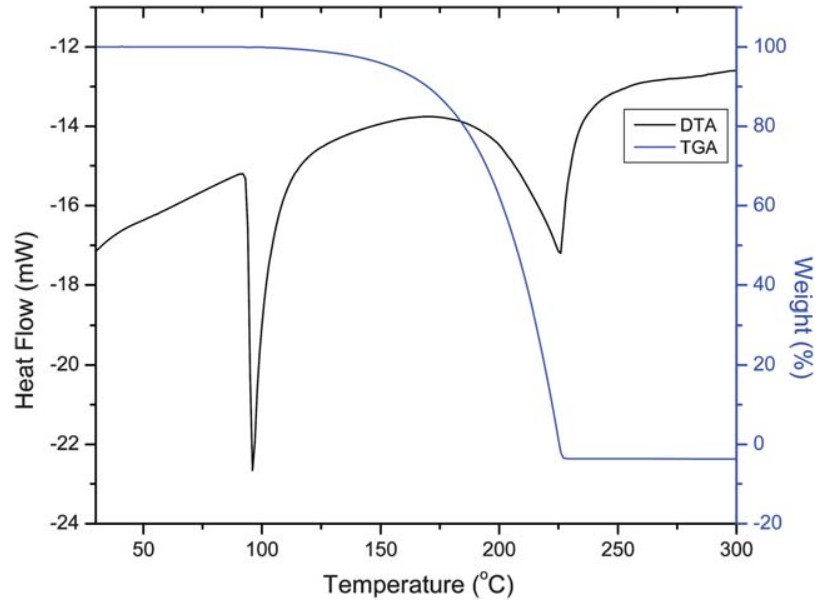

Fig. 6 TG/DTA spectrum of benzil.

\subsection{Optical transmission studies}

NLO single crystals are mainly used in optical applications, in which the optical transmission range and the transparency find an important place. The optical transmission spectra of single and double wall ampoules grown benzil crystals were recorded in the range 190-1100 $\mathrm{nm}$ using a Lambda 35 spectrophotometer. Cut and polished crystals of $2 \mathrm{~mm}$ thickness were used for the studies. Lan and Song ${ }^{9}$ have observed the cut-off wavelength at about $440 \mathrm{~nm}$ for the benzil crystal. UV-Vis-NIR spectra recorded for both samples are shown in Fig. 7. They show that both crystals have transparency greater than $60 \%$ with a cutoff wavelength of around $434 \mathrm{~nm}$. It is observed that the double wall ampoule grown crystal has a high transparency than the single wall ampoule grown crystal. In the growth with the double wall ampoule the temperature stress in the inner tube is less than that of the outer tube and for this reason the good crystal can be grown, hence the transmittance is increased. However, in the present study, we have observed an improvement in the optical transmittance when the single wall ampoule is replaced by the double wall ampoule. This can be attributed to the improvement in the control of thermal environments and the consequent

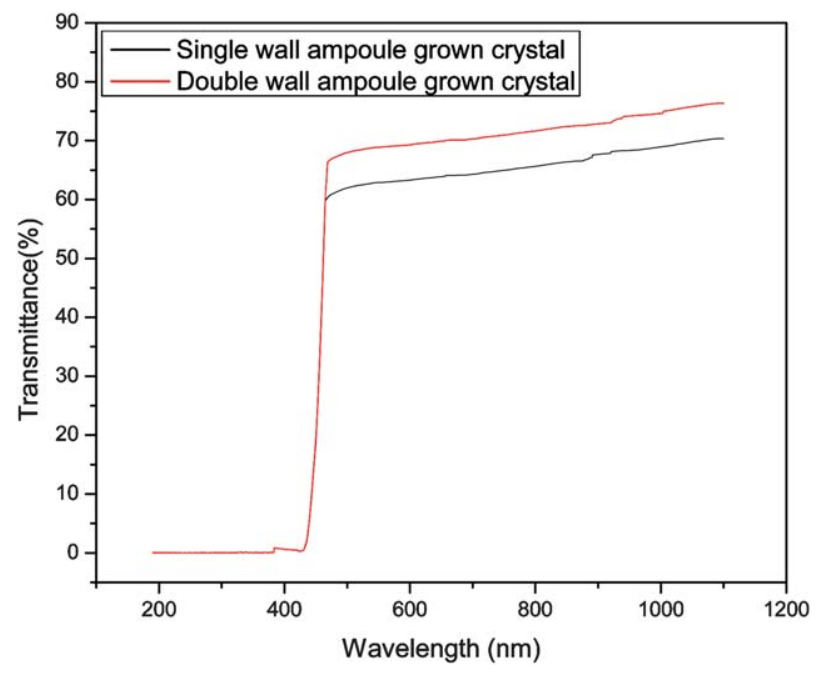

Fig. 7 UV-Vis-NIR spectra of benzil single crystals. 
improvement in the quality of the crystal grown when the double wall ampoule is used.

\subsection{Photoluminescence properties}

Photoluminescence spectra of the grown crystals were carried out by using the spectroflurometer with a $450 \mathrm{~W}$ high pressure Xenon lamp as an excitation source at room temperature. The grown crystals were excited at $398 \mathrm{~nm}$ and the emission spectra of single and double wall ampoules grown crystals are shown in Fig. 8(a) and (b). Both single and double wall ampoule grown crystals exhibit high intensity sharp peaks observed around $520 \mathrm{~nm}$. The results indicate that the benzil single crystal has a green emission. The green emission of benzil suggests that it may be used as a green light emitting material. Further, the double wall ampoule grown crystal exhibits high PL yield when compared to that observed for the single wall ampoule grown crystal.

\subsection{Dielectric studies}

Crystals with high transparency of size $7 \times 6 \times 2 \mathrm{~mm}^{3}$ were used for the dielectric measurements. The opposite faces were polished and coated with a high quality graphite to obtain a good conductive surface layer. The capacitance $\left(C_{\text {crys }}\right)$, air capacitance $\left(C_{\text {air }}\right)$ and dielectric loss factor $(\tan \delta)$ were measured using the
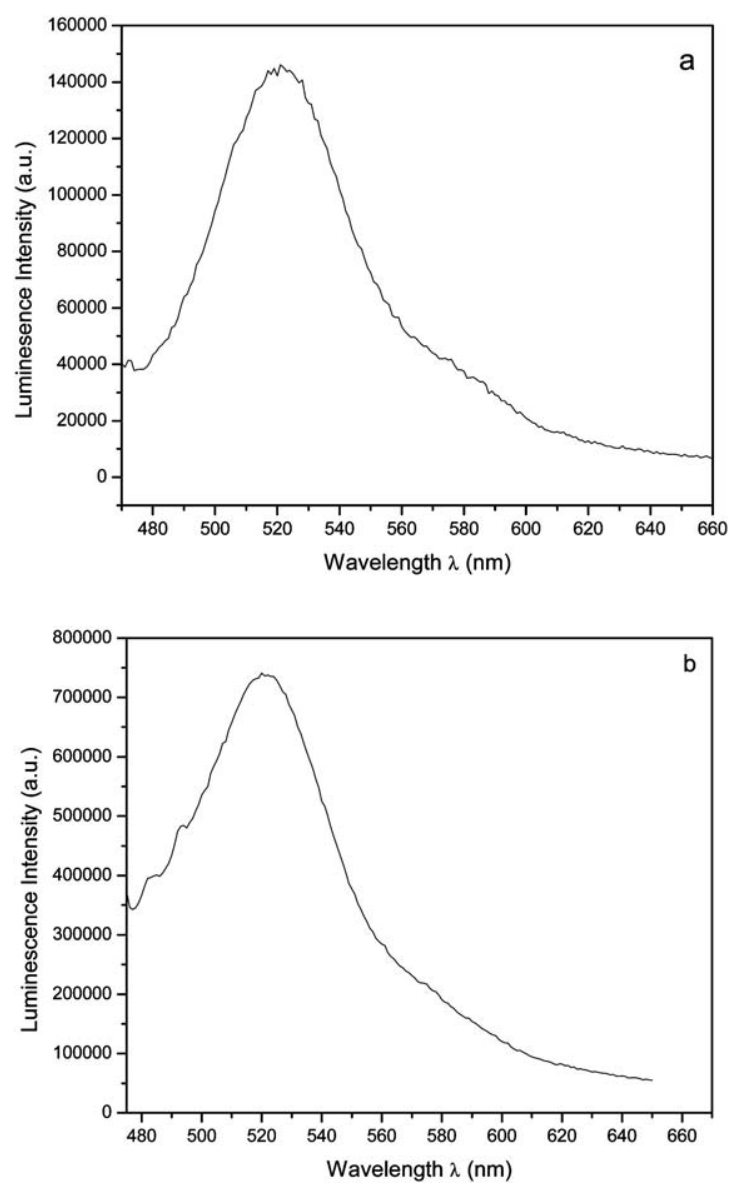

Fig. 8 (a) PL emission spectrum of the single wall ampoule grown benzil. (b) PL emission spectrum of the double wall ampoule grown benzil. conventional parallel plate capacitor method with four different frequencies, viz. $100 \mathrm{~Hz}, 1 \mathrm{kHz}, 10 \mathrm{kHz}$ and $100 \mathrm{kHz}$, using an Agilent 4284A LCR meter at various temperatures ranging from $313 \mathrm{~K}$ to $353 \mathrm{~K}$. The temperature was controlled to an accuracy of $\pm 1 \mathrm{~K}$. The observations were made while cooling the sample. The dielectric constant of the crystal was calculated using the relation

$$
\varepsilon_{\mathrm{r}}=\frac{C_{\text {crys }}}{C_{\text {air }}}
$$

The AC conductivity $\left(\sigma_{\mathrm{ac}}\right)$ was calculated using the relation:

$$
\sigma_{\mathrm{ac}}=\varepsilon_{\mathrm{o}} \varepsilon_{\mathrm{r}} \omega \tan \delta
$$

where $\varepsilon_{\mathrm{O}}$ is the permittivity of free space $\left(8.85 \times 10^{-12}\right.$ farad $\left.\mathrm{m}^{-1}\right)$ and $\omega$ is the angular frequency $(\omega=2 \pi f)$. Fig. 9(a)-(c) show the variation of dielectric constant $\left(\varepsilon_{\mathrm{r}}\right)$, dielectric loss $(\tan \delta)$ and $\mathrm{AC}$ conductivity $\left(\sigma_{\mathrm{ac}}\right)$ observed for the benzil crystal grown with the double wall ampoule.

It can be seen that the dielectric parameters, viz. dielectric constant $\left(\varepsilon_{\mathrm{r}}\right)$, dielectric loss $(\tan \delta)$ and AC conductivity $\left(\sigma_{\mathrm{ac}}\right)$, increase with the increase in temperature. The dielectric constant $\left(\varepsilon_{\mathrm{r}}\right)$ and dielectric loss $(\tan \delta)$ values decrease with the increase in frequency while the AC conductivity $\left(\sigma_{\mathrm{ac}}\right)$ value increases with the increase in frequency. This is a normal dielectric behaviour. This can be understood on the basis that the mechanism of polarization is similar to the conduction process. The electronic exchange of the number of ions in the crystal gives local displacement of electrons in the direction of the applied field, which in turn gives rise to polarization. The high value of dielectric constant at low frequencies may be associated with space charge, dipolar, electronic and ionic polarization and its low value at higher frequencies may be due to the loss of significance of these polarizations gradually. Space charge polarization is generally active at lower frequencies and higher temperatures and indicates the perfection of crystals. The low value of dielectric loss at high frequency implies that the crystal possesses good optical quality with lesser defects and this parameter is of vital importance for NLO materials in their applications. $^{23}$

Several innovative developments have been made for the development of new low $\varepsilon_{\mathrm{r}}$ value materials to replace silica $\left(\varepsilon_{\mathrm{r}} \approx\right.$ 4.0). However, there is still a need for new low $\varepsilon_{\mathrm{r}}$ value dielectric materials. As the utility in the electronic circuits with water proof conditions, water soluble materials in the single crystal form would be very much interesting. Recently, Mahadevan and his coworkers ${ }^{24,25}$ have reported reduction in $\varepsilon_{\mathrm{r}}$ value in the case of potassium dihydrogen orthophosphate (KDP) added with urea and L-arginine. Also, L-arginine acetate (LAA) and L-arginine oxalate (LAO) ${ }^{26}$ have been found to be promising low $\varepsilon_{\mathrm{r}}$ value dielectric materials. Fig. 9(a) indicates that the $\varepsilon_{\mathrm{r}}$ values observed for the benzil crystal in the present study are low, $<4.0$ up to $323 \mathrm{~K}$ for all the four frequencies considered. Low $\varepsilon_{\mathrm{r}}$ values have been observed for higher frequencies even at high temperatures. This indicates that the benzil crystal grown in the present study can be considered as a promising low $\varepsilon_{\mathrm{r}}$ value dielectric material. The present study, in effect, indicates that the grown benzil single crystal is not only a potential NLO material but also a promising low $\varepsilon_{\mathrm{r}}$ value dielectric material. 

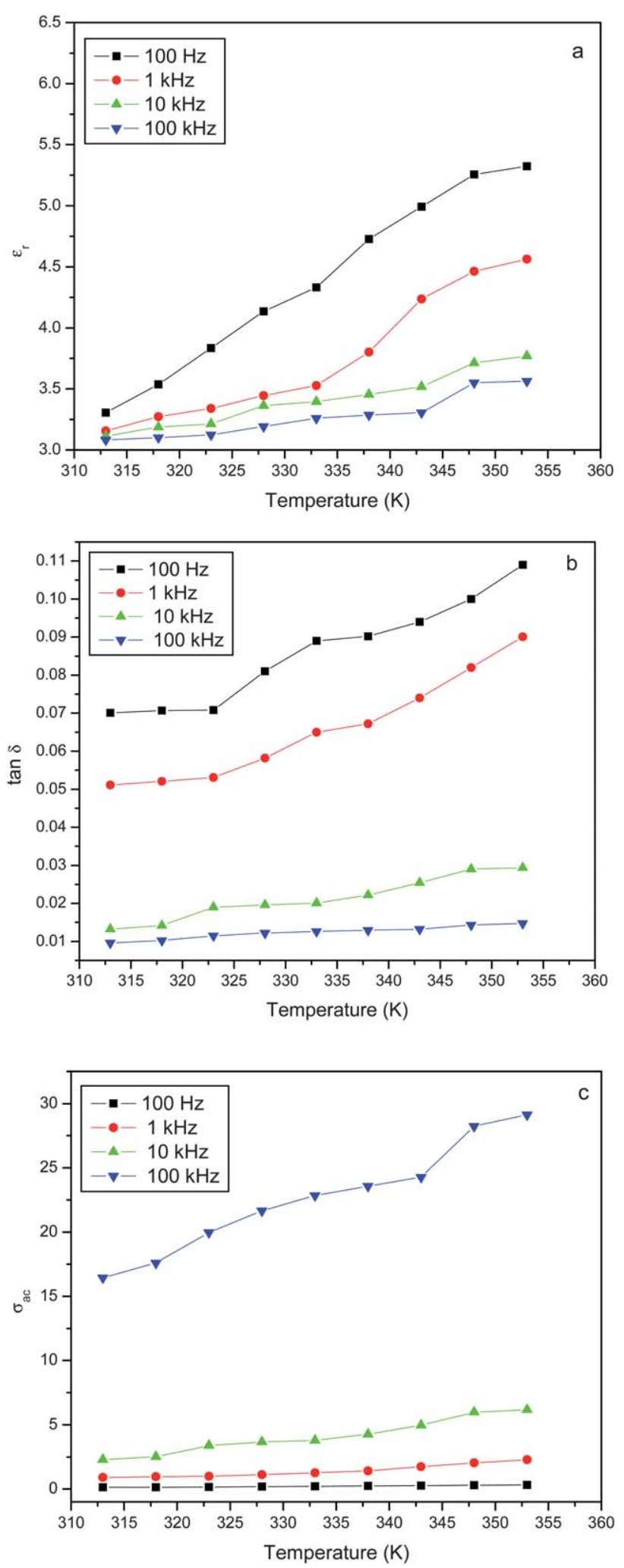

Fig. 9 Double wall grown benzil crystal: (a) plot of dielectric constant versus temperature, (b) plot of dielectric loss versus temperature and (c) plot of AC electrical conductivities $\left(\times 10^{-8} \mathrm{mho}^{-1}\right)$ versus temperature.

A comparison of $\varepsilon_{\mathrm{r}}$ and $\tan \delta$ values observed with $1 \mathrm{kHz}$ frequency is done in Fig. 10(a) and (b) for benzil crystals grown using single and double wall ampoules. Replacement of the single wall ampoule with the double wall ampoule increases the $\varepsilon_{\mathrm{r}}$ value and decreases the $\tan \delta$ value. This also confirms that the quality of the benzil crystals has improved when grown using the double wall ampoule.

\subsection{NLO studies}

SHG conversion efficiency of the grown benzil crystal sample was measured by the Kurtz and Perry technique. Nd:YAG $(\lambda=$ $1064 \mathrm{~nm}$ ) laser irradiates the powder sample of benzil kept in the triangular cell. The monochromator was set at $532 \mathrm{~nm}$. The NLO signal was captured by the oscilloscope through the photomultiplier tube. The Nd:YAG laser source produces nanosecond pulses ( $8 \mathrm{~ns}$ ) of $1064 \mathrm{~nm}$ light and the energy of the laser pulse was around $30 \mathrm{~mJ}$. The beam emerging through the sample was focused onto a Czerny-Turner monochromator using a pair of lenses. The detection was carried out using a Hamamatsu R928 photomultiplier tube. The signals were captured with an Agilent infinium digital storage oscilloscope interfaced to a computer. The generation of the second harmonic was confirmed by the emission of green light. Urea sample was used as the reference
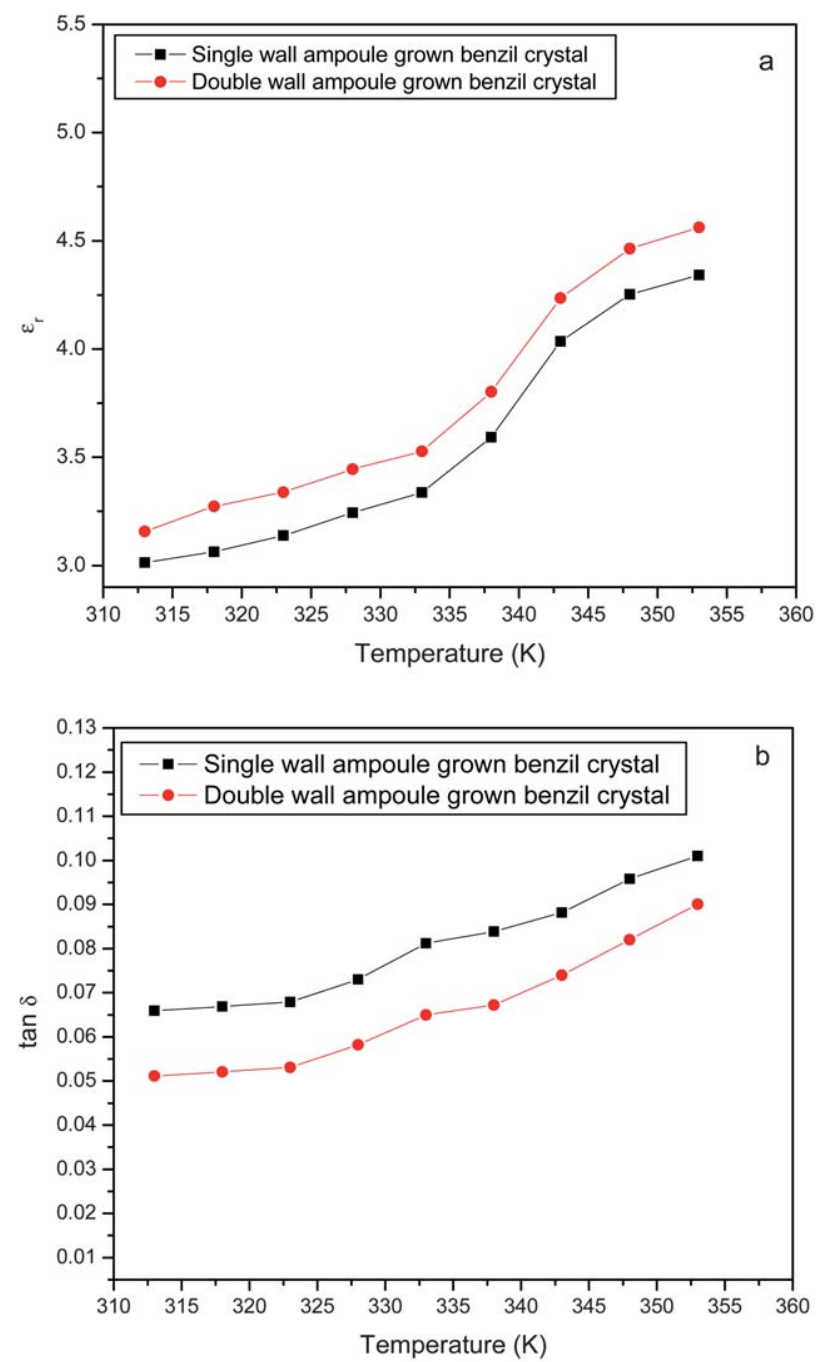

Fig. 10 (a) Dielectric constants with $1 \mathrm{kHz}$ frequency compared and (b) dielectric losses with $1 \mathrm{kHz}$ frequency compared. 
material in the SHG measurements. The result reveals that the SHG efficiency of benzil is 0.64 times than that of urea.

\section{Conclusions}

Benzil single crystals were grown by the modified vertical Bridgman technique with nanotranslation using single and double wall ampoules. The grown benzil crystals were confirmed by single crystal XRD, powder XRD and FTIR analysis. HRXRD analysis reveals that the crystalline perfection was reasonably good. Thermal studies reveal that the grown crystal melts at $96{ }^{\circ} \mathrm{C}$ and below its melting point no phase transformation occurs. UV-Vis-NIR studies show that the grown crystals have good transparency in the visible and near IR regions. The photoluminescence spectrum of benzil crystals indicates green emission. The SHG analysis reveals that the efficiency of benzil is 0.64 times higher than that of urea. Dielectric measurements show that dielectric constant, dielectric loss and AC conductivity values increase with the increase of temperature which can be understood as due to the temperature variation of electronic polarizability. The results obtained in the present study indicate that the grown benzil single crystal is not only a potential NLO material but also a promising low dielectric constant value dielectric material, expected to be useful in the microelectronics industry. In addition, the present study indicates that the benzil crystal grown with the double wall ampoule is superior to that grown with the single wall ampoule.

\section{Acknowledgements}

This work, supported by the Department of Science and Technology, New Delhi, India under the grant of project ref-SR/FTP/ PS-20/2005, is hereby gratefully acknowledged. The authors thank Prof. P. Ramamurthy, National Centre for Ultrafast Processes, University of Madras, Chennai for extending the SHG studies and Dr U. Madhusoodhanan, Indira Gandhi Center for Atomic Research, Kalpakkam for support in photoluminescence studies and for his valuable suggestions and useful discussions.

\section{References}

1 J. Thompson, R. I. R. Blyth, M. Mazzeo, M. Anni, G. Gigli and R. Cingoloni, Jpn. J. Appl. Phys., Part 2, 2001, 79, 560-562.

2 X. T. Tao, D. R. Yuan, N. Zang, M. H. Jiang and Z. S. Shao, Jpn. J. Appl. Phys., Part 2, 1992, 60, 1415-1417.

3 P. Gunter, Nonlinear Optical Effects and Materials, Springer, Berlin, 2000 .

4 M. S. Wong, C. Bosshard, F. Pan and P. Gunter, Adv. Mater., 1996, 8, 677-680.

5 S. Natarajan, S. A. Martin Britto and E. Ramachandran, Cryst. Growth Des., 2006, 6, 137-140.

6 B. D. Hatton, K. Landskron, W. J. Hunks, M. R. Bennett, D. Shukaris, D. D. Perovic and G. A. Ozin, Mater. Today, 2006, 9, 22-31.

7 R. N. Rai and K. B. R. Varma, Mater. Lett., 2001, 48, 356-361.

8 Th. Scheffen-Lauenroth, H. Klapper and R. A. Becker, J. Cryst. Growth, 1981, 55, 557-570.

9 C. W. Lan and C. R. Song, J. Cryst. Growth, 1997, 180, 127-135.

10 J. Bleay, R. M. Hooper, R. S. Narang and J. N. Sherwood, J. Cryst. Growth, 1978, 43, 589-596.

11 M. D. Aggarwal, W. S. Wang and M. Tambwe, Mater. Res. Bull., 1992, 27, 431-437.

12 M. D. Aggarwal, W. S. Wang and M. Tambwe, J. Cryst. Growth, 1993, 128, 891-896.

13 M. V. Shankar and K. B. R. Varma, Bull. Mater. Sci., 1996, 19, 791798.

14 T. Suthan, N. P. Rajesh, P. V. Dhanaraj and C. K. Mahadevan, Spectrochim. Acta, Part A, 2010, 75, 69-73.

15 N. K. Udayashankar and H. L. Bhat, Bull. Mater. Sci., 2001, 24, 445453.

16 N. Balamurugan, A. Arulchakkaravarthi and P. Ramasamy, J. Cryst. Growth, 2008, 310, 2115-2119.

17 A. Arulchakkaravarthi, P. Santhanaraghavan and P. Ramasamy, $J$. Cryst. Growth, 2001, 224, 89-94.

18 P. Mohan, N. Senguttuvan, S. Moorthy Babu and P. Ramasamy, $J$. Cryst. Growth, 2000, 211, 207-210.

19 JCPDS file card no: $30-1539$.

20 R. M. Silverstein, G. C. Bassler and T. C. Morrill, Spectrometric Identification of Organic Compounds, Wiley, New York, 1991.

21 K. Lal and G. Bhagavannarayana, J. Appl. Crystallogr., 1989, 22, 209-215.

22 G. Bhagavannarayana, R. V. Ananthamurthy, G. C. Budakoti, B. Kumar and K. S. Bartwal, J. Appl. Crystallogr., 2005, 38, 768-771.

23 K. V. Rao and A. Smakula, J. Appl. Phys., 1965, 36, 2031-2038.

24 S. Goma, C. M. Padma and C. K. Mahadevan, Mater. Lett., 2006, 60, 3701-3705.

25 M. Meena and C. K. Mahadevan, Cryst. Res. Technol., 2008, 43, 166172.

26 M. Meena and C. K. Mahadevan, Mater. Lett., 2008, 62, 3742-3744. 